\title{
A Mathematical Model for Predicting Rate of Divorce Tendency in Nigeria: A Study of Taraba State, Nigeria
}

\author{
Ogwumu, Onah.David \\ Department of Mathematics and Statistics, Federal University Wukari, Nigeria \\ Corresponding Author: onahdavid2010@gmail.com, davidonah@fuwukari.edu.ng
}

\author{
Kyagya T.Yusuf, Amakoromo Grace.I., Keto, Kingsley M., Ezeh A.Tochukwu., Ogofotha Marvellous \\ O., Elugah Joseph.I. \\ Department of Mathematics and Statistics, Federal University Wukari, Nigeria \\ E-mail:tizhetizhekyagya@gmail.com,dabtgrace@yahoo.com,kings.keto5@gmail.com, \\ amoscotk0@gmail.com, marvellousovuzorie@gmail.com, joecalculus1@gmail.com
}

Received: 10 May 2020; Accepted: 15 July 2020; Published: 08 October 2020

\begin{abstract}
Given that divorce has become a recurring challenge with increasing intensity in our world today accompanying broken families, economy and social contagion, as well as the existence of difference in happiness between the couple and their children. Thus, a mathematical model for predicting the rate of divorce tendencies in the Nigerian society is hereby developed. Factors influencing the rate of divorce were outlined and mathematical relationships between these factors were established. Afterwards, the developed model was validated and the real life data collected were contrasted with the model data predictions using suitable statistical tools. The findings from the comparison showed that the real life data and the model data predictions have a higher degree of correlation; consequently, recommending the model as a benchmark measure for predicting rate of divorce/marital instability in Nigeria. In the same vein, recommendations were made at the end of the model analysis which when adhered to would yield.
\end{abstract}

Index Terms: rate of divorce, model prediction, Income Difference parameter, Educational Difference parameter, Age Difference parameter, Least Squares Method.

\section{Introduction}

Divorce rate in Africa and Nigeria to be specific, is worth giving attention to if we are really interested in having a great continent. This is because; divorce has a higher influence on the productivity and performance of any nation. Besides, in order to be able to achieve the 16th goal of the SDGs that advocates for peace and justice in our society, increasing divorce rate has to be considerably managed in our African society.

According to the National Bureau of Statistics, official statistics of the nation Nigeria suggested that divorce is exceedingly uncommon in Nigeria. Just $0.2 \%$ of men and $0.3 \%$ of women have legally untied the knot. Meanwhile, less than $1 \%$ of couples admitted the status of being separated [2]. Yet such counts exclude the vast majority of Nigerians, whose traditional marriage ceremonies are not governed by modern law, says Chief Robert Clarke, a barrister.

"Divorce is a delicate accident in marital relationships. What constitutes a divorce must be viewed against the fact that marriage is a process. It should be such that once the full contract of marriage has been executed, it is extremely hard to dissolve it'"[4].

In the words of [9], "Divorce introduces a massive change into the lives of the children whether boy or girl no matter what the age may be. Witnessing loss of love between parents, having parents break their marriage commitment, adjusting to going back and forth between two different households, and the daily absence of one parent while living with the other, all create a challenging new family circumstance in which to live". In the personal history of the boy or girl, parental divorce is a watershed event. Life that follows is significantly changed from how life was before. Meanwhile in Nigeria, According to [1], the forms of divorces common in Nigeria and most African locals are: Voluntary or Walk-Away Divorce, Military or Forceful Divorce and Family-Resolved Divorce.

Although researchers haveargued that another type of divorce called informal divorce has the highest percentage so far. Meanwhile, [5]remarked that Informal Divorce this is when couples live apart but do not pursue formal separation or 
divorce. It could be thought of as a trial separation. Whichever form of divorce anyone embarks on, the standpoint of [6] is that divorce and its associated implications directly impact on individuals, society and the nation at large

Consequently, divorce shakes trust in dependency on parents for the young child as they (parents) now behave in an extremely undependable way. They surgically divide the family unit into two different households between which the child must learn to transit back and forth, for a while creating unfamiliarity, instability, and insecurity, never being able to be with one parent without having to be apart from the other [4]. He further stated, convincing a young child of the permanence of divorce can be hard when his intense longing daydreams remain that somehow, some way, mom and dad will be living back together again someday. He relies on wishful thinking to help allay the pain of loss, holding onto hope for a parental reunion much longer than does the adolescent who is quicker to accept the finality of this unwelcome family change. Thus parents who put in a joint presence at special family celebrations and holiday events to recreate family closeness for the child only feed the child's fantasy and delay his adjustment.

According to [3], divorce is a legal or customary decree that a marriage is dissolved, in other words, divorce is a permanent separation of married people as a result of unexpected marriage outcome. The increasing divorce rate in the world today prompted the need to mathematically examine the various factors that causes divorce. Researchers like; [10], used a non-linear MSD mathematical model to study the dynamics of divorce epidemic while [11] is of the opinion that divorces are related to the economy and social contagion. Hence, in this research we proposed a deterministic model that incorporates major factors that influences divorce.

Researchers like $[12,13,14,15]$ outlined the effects of divorce on children; use of illicit substances, low academic achievement, be more likely to have a teen pregnancy, suffer from social isolation, exhibit externalizing and internalizing behaviour problems, fall victim to adult mental health problems, have low social responsibility and diminished achievement, among others. It is hoped that results from this study will enrich couples with modalities in handling variables leading to divorce in marriages. Secondly, this research provides users with the model that can forecast point(s) of divorce in marriages. Likewise, the mathematical model proposed in this study is intended for reduction of the current divorce rate in the country. The study will also save the Nigerian child from the possibility of poor performance in school as well as our country from challenges resulting from broken homes.

\section{LITERATURE REVIEW}

From the definition of marriage it is almost impossible to exhaustively discuss the topic divorce in Nigeria without first of all, considering marriage situations in the country. This is because, divorce is a concurrence of marriage and there cannot be divorce without marriage. According to [6], "Marriage is a social union or legal contract that establishes rights and obligations between the spouses, between spouses and their children, and between the spouses and their in-laws".

Likewise, [16], stated that marriage is an institution which was established by God. It is sacred and it is a lifelong commitment as commanded by God and not a death sentence like some think it is. Christianity and a few other religions emphasize on the sanctity of marriage, be it monogamy or polygamy. "Thus it is for this cause that a man shall leave his mother and clings to his wife and together they become one" [7]. Relating to this, they (couples) stand before friends and loved ones and declare their love for each other taking vows that uphold that they would stay together in sickness and in health and through all other unforeseen situations until death do them part. (This implies that they know it is not going to be all rosy to the end). Together they start a family and make their home.

Divorce in Nigeria is not always a fun topic; in fact, most people shy away from it and perceive that divorce as a taboo subject matter. Unfortunately, it is the reality of the world we live in that some couples after getting married will decide for whatever reason that they do not want to carry on with their marriage and want a divorce. This has been position and grounds for divorce in Nigeria thus far [17].

\section{METHODOLOGY}

In this chapter, we shall consider the following subheadings as they unfold:

\subsection{Formulation of Model Equation}

This section will address a few subtopics as they unfold.

\subsubsection{Divorce Rate Versus Age Difference}

If the "age difference" A, of married couples is low the chances are that divorce tendencies will be low or zero, and when their age difference is high the tendencies of divorce D, will be high as well. Hence, when an aged man marries an underage lady (and vice versa), then the possibility for divorce is almost inevitable. Thus, based on the above assertion, we can remark that the tendencies for couples suing for a divorce $\mathrm{D}$, is directly proportional to their age difference A. this can be represented Mathematically as; 


$$
\begin{array}{r}
D \propto A \\
\Rightarrow D=k_{1} A
\end{array}
$$

\subsubsection{Divorce Rate (D) Versus Educational Difference (E)}

Again, the lower the "educational difference" E, of any set of married couples, the lesser or nil chances for divorce D, to exist. It follows vice versa for when their educational difference is high. Hence, the educational difference E, of any set of married couples is directly proportional to its divorce rate. Hence, mathematically;

$$
\begin{array}{cc} 
& D \propto E \\
\Rightarrow & D=k_{2} E
\end{array}
$$

\subsubsection{Divorce Rate Versus Income (ID) Difference}

In this case, when the "income difference" Id, of married couples is low the less it generates tendencies for divorce and the same applies to when income difference is high which leads to greater tendencies of divorce D. Hence, the income difference $\mathrm{I}_{\mathrm{d}}$, of any married couples is directly proportional to divorce. This case often relies on whether the high earnings are that of the man or woman. Hence, mathematically represented as;

$$
\begin{gathered}
\quad D \propto I_{D} \\
\Rightarrow \quad D=k_{3} I_{D}
\end{gathered}
$$

\subsubsection{Divorce Rate versus Total income (IT) of couples}

In this relationship, when the total income $\left(\mathrm{I}_{\mathrm{T}}\right)$ of the family is high or normal, there will be minimal or no divorce development in the marriage. Conversely, there is likelihood of high divorce development in the same marriage if the total earnings of couples are lower than their demands. This implies that the total income $\left(\mathrm{I}_{\mathrm{T}}\right)$ of a man and his wife is inversely proportional to divorce rate (D). Mathematically given as;

$$
\begin{aligned}
D & \propto \frac{1}{I_{T}} \\
\Rightarrow \quad D & =\frac{k_{4}}{I_{T}}
\end{aligned}
$$

\subsubsection{General Establishment of Model Parameters Relationship}

From the parameter equations constructed above, if we add (3.1), (3.2), (3.3) and (3.4) we would have:

$$
\begin{aligned}
& D=k_{1} A \\
& D=k_{2} E \\
& \left.D=k_{3} I_{d}\right\} \text { Adding gives; } \\
& D=\frac{k_{4}}{I_{T}} \\
& 4 D=k_{1} A+k_{2} E+k_{3} I_{d}+\frac{k_{4}}{I_{T}} \\
& \Rightarrow D=a A+b E+c I_{D}+\frac{d}{I_{T}}
\end{aligned}
$$

Given that; $a=\frac{k_{1}}{4}, b=\frac{k_{2}}{4}, c=\frac{k_{3}}{4}, d=\frac{k_{4}}{4}$

Where; $\mathrm{D}=$ Divorce rate; $\mathrm{A}=$ Age difference; $\mathrm{I}_{\mathrm{D}}=$ Income difference; $\mathrm{E}=$ Educational difference.

$\mathrm{I}_{\mathrm{T}}=$ Total income and $\mathrm{a}, \mathrm{b}, \mathrm{c}$ and $\mathrm{d}$ are the model constants.

3.1.6 Assumptions Based on the Mathematical Behaviour of the Parameters Relationship. 
Under this heading, the nature of equation view was given to the relationship that existed between the variables of the model. From the relationship graph pre-plotted for divorce rate, against couple's age difference, educational difference and income differences, the following equations regarding each factor versus divorce rate were observed. And hence from the emerging equations;

i. We assume that the relationship between divorce D and age difference A, is linear,

$$
D=a^{\prime} A+t_{1}
$$

ii. Similarly, the relationship between $\mathrm{D}$, and education $\mathrm{E}$, is linear,

$$
D=b^{\prime} E+t_{2}
$$

iii. And the relationship between divorce and income difference is non-linear, so, let its nature of equation be,

$$
D=c^{\prime} I_{D}+t_{3}
$$

iv. More so, the relationship between the total income of couples and divorce is a non-linear one. Hence, its type of equation is;

$$
D=\frac{d^{\prime}}{I_{T}}+t_{4}
$$

Where $D, A, E, I_{D}$ and $I_{T}$ remain the usual definitions as above, and $t_{1}, t_{2}, t_{3}$ and $t_{4}$ are constants

\subsection{Second Establishment of Model Parameters Relationships.}

Adding equation (6), (7), (8) and (9) yields,

$$
\begin{gathered}
4 D=\left(a+a^{\prime}\right) A+\left(b+b^{\prime}\right) E+\left(c+c^{\prime}\right) I_{D i}+\frac{\left(d+d^{\prime}\right)}{I_{T}}+\left(t_{1}+t_{2}+t_{3}+t_{4}\right) \\
D=\frac{\left(a+a^{\prime}\right) A}{4}+\frac{\left(b+b^{\prime}\right) E}{4}+\left(c+c^{\prime}\right) I_{D}+\frac{\left(d+d^{\prime}\right)}{4 I_{T}}+\frac{\left(t_{1}+t_{2}+t_{3}+t_{4}\right)}{4} \\
\therefore D=x A+y E+z I_{D i}+\frac{u}{I_{T}}+v
\end{gathered}
$$

Where: $\mathrm{D}=$ Divorce rate

$A=$ Age difference

$E=$ Educational difference

$I_{D}=$ Income difference

$\mathrm{I}_{\mathrm{T}}=$ Total income of couples

And $x, y, z, u$ and $v$ are the model constants.

\subsection{Evaluating the Model Equation Constants.}

To evaluate the constants in the model above, equation (11) is been differentiated partially with respect to $\mathrm{x}, \mathrm{y}, \mathrm{z}, \mathrm{u}$ and $\mathrm{v}$ respectively following the Least Squares Method of obtaining model equation constants.

Minimizing the propose model in equation (11) using least squares method, we obtain the results below:

$$
Z_{\text {Min }}=\min \sum\left(D_{i}-x A_{i}-y E_{i}-z I_{d i}-\frac{u}{I_{T i}}-v\right)^{2}, \forall=1,2, . ., n \text {. }
$$

At, optimal point, $\frac{\partial Z}{\partial x}=\frac{\partial Z}{\partial y}=\frac{\partial Z}{\partial z}=\frac{\partial Z}{\partial u}=\frac{\partial Z}{\partial v} 0$.

Hence, differentiating (12) partially with respect to each of the constants, $x, y, z, u$ and $v$ gives,

$$
\frac{\partial Z}{\partial x}=-2 \sum_{i=1}^{n}\left(D_{i}-x A_{i}-y E_{i}-z I_{d i}-\frac{u}{I_{T i}}-v\right) A_{i}=0
$$




$$
\begin{aligned}
& \frac{\partial Z}{\partial y}=-2 \sum_{i=1}^{n}\left(D_{i}-x A_{i}-y E_{i}-z I_{d i}-\frac{u}{I_{T i}}-v\right) E_{i}=0 \\
& \frac{\partial Z}{\partial z}=-2 \sum_{i=1}^{n}\left(D_{i}-x A_{i}-y E_{i}-z I_{d i}-\frac{u}{I_{T i}}-v\right) \frac{1}{I_{D i}}=0 \\
& \frac{\partial Z}{\partial u}=-2 \sum_{i=1}^{n}\left(D_{i}-x A_{i}-y E_{i}-z I_{d i}-\frac{u}{I_{T i}}-v\right) \frac{1}{I_{T i}}=0 \\
& \frac{\partial Z}{\partial v}=-2 \sum_{i=1}^{n}\left(D_{i}-x A_{i}-y E_{i}-z I_{d i}-\frac{u}{I_{T i}}-v\right)=0
\end{aligned}
$$

Further simplification of equations (13)-(17) yields,

$$
\begin{gathered}
\sum_{i=1}^{n} D_{i} A_{i}=x \sum_{i=1}^{n} A_{i}^{2}+y \sum_{i=1}^{n} A_{i} E_{i}+z \sum_{i=1}^{n} A_{i} I_{D i}+u \sum_{i=1}^{n} \frac{A}{I_{T i}}+v \sum_{i=1}^{n} A_{i}=0 \\
\sum_{i=1}^{n} D_{i} E_{i}=x \sum_{i=1}^{n} A_{i}+y \sum_{i=1}^{n} E_{i}^{2}+z \sum_{i=1}^{n} E_{i} I_{D i}+u \sum_{i=1}^{n} \frac{E_{i}}{I_{T i}}+v \sum_{i=1}^{n} E_{i}=0 \\
\sum_{i=1}^{n} D_{i} I_{D i}=x \sum_{i=1}^{n} A_{i} I_{D i}+y \sum_{i=1}^{n} E_{i} I_{D i}+z \sum_{i=1}^{n} I_{D i}^{2}+u \sum_{i=1}^{n} \frac{I_{D i}}{I_{T i}}+v \sum_{i=1}^{n} I_{D i}=0 \\
\sum_{i=1}^{n} \frac{D_{i}}{I_{T i}}=x \sum_{i=1}^{n} \frac{A_{i}}{I_{T i}}+y \sum_{i=1}^{n} \frac{E_{i}}{I_{T i}}+z \sum_{i=1}^{n} \frac{I_{D i}}{I_{T i}}+u \sum_{i=1}^{n} \frac{1}{I_{T i}^{2}}+v \sum_{i=1}^{n} \frac{1}{I_{T i}}=0 \\
\sum_{i=1}^{n} D_{i}=x \sum_{i=1}^{n} A_{i}+y \sum_{i=1}^{n} E_{i}+z \sum_{i=1}^{n} I_{D i}+u \sum_{i=1}^{n} \frac{1}{I_{T i}}+v \sum_{i=1}^{n} n=0
\end{gathered}
$$

The next task here is to gather data in order to evaluate our Model equation constants as given in the succeeding section.

\subsection{Research Instrument Used}

The investigation instrument used for this work is known as purposeful sampling technique. This is a situation where a certain sample of Taraba state Nigeria's indigence was decisively selected based on some features and the objectives of the research. However, during the study, only the questionnaires retrieved from the set of persons that are not singles, widows or widowers were considered. Such status as stated above may affect the authenticity of our research model and may not allow the model results to conform to reality. Also, the data collected were appropriate as they were directly from the affected persons. After all possible and positive screening of the data, some selected set of data properly filled on the distributed questionnaires were finally considered for the research. 
Table 1. Family welfare Data gathered for the Research.

\begin{tabular}{|c|c|c|c|c|}
\hline S/N & Age difference A. & Education difference $\mathbf{E}$. & Income difference $\mathbf{I}_{\mathbf{D}} \cdot$ & Total income $\mathbf{I}_{\mathbf{T}}$ \\
\hline 1 & 4 & 0 & 20,000 & 110,000 \\
\hline 2 & 3 & 0 & 1,000 & 13,000 \\
\hline 3 & 8 & 1 & 13,000 & 23,000 \\
\hline 4 & 5 & 1 & 10,000 & 130,000 \\
\hline 5 & 12 & 1 & 7,000 & 23,000 \\
\hline 6 & 8 & 1 & 24,800 & 25,200 \\
\hline 7 & 4 & 1 & 2,000 & 38,000 \\
\hline 8 & 14 & 1 & 13,000 & 27,000 \\
\hline 9 & 10 & 1 & 15,000 & 105,000 \\
\hline 10 & 3 & 0 & 55,000 & 60,000 \\
\hline 11 & 7 & 0 & 15,000 & 45,000 \\
\hline 12 & 8 & 0 & 20,000 & 210,000 \\
\hline 13 & 3 & 0 & 10,000 & 85,000 \\
\hline 14 & 4 & 1 & 10,000 & 50,000 \\
\hline 15 & 7 & 1 & 10,000 & 70,000 \\
\hline 16 & 3 & 0 & 20,000 & 60,000 \\
\hline 17 & 7 & 1 & 5,000 & 35,000 \\
\hline
\end{tabular}

Source: field questionnaire data collected from Taraba State, Nigerian dwellers

\subsection{Evaluation of the Equation Constants Using the Data in Table 1.}

Table 2.Multiplication Results of the Figures in Table 1 above

\begin{tabular}{|c|c|c|c|c|c|c|}
\hline $\mathbf{A}$ & $\mathbf{E}$ & $\mathbf{I}_{\mathbf{D}}$ & $\mathbf{I}_{\mathbf{T}}$ & Divorce $\mathbf{D}$ & $\mathrm{A}^{2}$ & $\mathbf{E}^{2}$ \\
\hline 4 & 0 & 20,000 & 110,000 & 0.1 & 16 & 0 \\
\hline 3 & 0 & 1,000 & 13,000 & 0.5 & 9 & 0 \\
\hline 8 & 1 & 13,000 & 23,000 & 0.4 & 64 & 1 \\
\hline 5 & 1 & 10,000 & 130,000 & 0.1 & 25 & 1 \\
\hline 12 & 1 & 7,000 & 23,000 & 0.2 & 144 & 1 \\
\hline 8 & 1 & 24,800 & 25,200 & 0.4 & 64 & 1 \\
\hline 4 & 1 & 2,000 & 38,000 & 0.3 & 16 & 1 \\
\hline 14 & 1 & 13,000 & 27,000 & 0.2 & 196 & 1 \\
\hline 10 & 1 & 15,000 & 105,000 & 0.1 & 100 & 1 \\
\hline 3 & 0 & 55,000 & 60,000 & 0.25 & 9 & 0 \\
\hline 7 & 0 & 15,000 & 45,000 & 0.15 & 49 & 0 \\
\hline 8 & 0 & 20,000 & 210,000 & 0.05 & 64 & 0 \\
\hline 3 & 0 & 10,000 & 85,000 & 0.1 & 9 & 0 \\
\hline 4 & 1 & 10,000 & 50,000 & 0.15 & 16 & 1 \\
\hline 7 & 1 & 10,000 & 70,000 & 0.05 & 49 & 1 \\
\hline 3 & 0 & 20,000 & 60,000 & 0.25 & 9 & 0 \\
\hline 7 & 1 & 5,000 & 35,000 & 0.25 & 49 & 1 \\
\hline \multirow{2}{*}{$\sum_{i=1}^{17} \mathbf{A}=$} & \multirow{2}{*}{$\sum_{i=1} E=$} & $\sum_{i=1}^{17} I_{D}$ & $\sum_{i=1}^{17} I_{T}=$ & $\sum_{i=1} D=$ & $\sum_{i=1} \mathbf{A}^{2}=$ & $\sum_{i=1} E^{2}=$ \\
& & 250800 & 11092000 & 3.55 & 888 & 10 \\
\hline
\end{tabular}


Table 3. Multiplication Results of the Figures in Table 1 above (Continuation)

\begin{tabular}{|c|c|c|c|c|c|c|}
\hline$\left(\mathbf{I}_{\mathrm{D}}\right)^{2}$ & A*E & $A * D$ & $\mathrm{D} * \mathrm{E}$ & $\mathrm{A}^{*} \mathbf{I}_{\mathbf{D}}$ & $\mathrm{E}^{*} \mathbf{I}_{\mathbf{D}}$ & $\mathrm{D}^{*} \mathbf{I}_{\mathbf{D}}$ \\
\hline 400000000 & 0 & 0.4 & 0 & 80000 & 0 & 2000 \\
\hline 1000000 & 0 & 1.5 & 0 & 3000 & 0 & 500 \\
\hline 169000000 & 8 & 3.2 & 0.4 & 104000 & 13000 & 5200 \\
\hline 100000000 & 5 & 0.5 & 0.1 & 50000 & 10000 & 1000 \\
\hline 49000000 & 12 & 2.4 & 0.2 & 84000 & 7000 & 1400 \\
\hline 615040000 & 8 & 3.2 & 0.4 & 198400 & 24800 & 9920 \\
\hline 4000000 & 4 & 1.2 & 0.3 & 8000 & 2000 & 600 \\
\hline 169000000 & 14 & 2.8 & 0.2 & 182000 & 13000 & 2600 \\
\hline 225000000 & 10 & 1 & 0.1 & 150000 & 15000 & 1500 \\
\hline 3025000000 & 0 & 0.75 & 0 & 165000 & 0 & 13750 \\
\hline 225000000 & 0 & 1.05 & 0 & 105000 & 0 & 2250 \\
\hline 400000000 & 0 & 0.4 & 0 & 160000 & 0 & 1000 \\
\hline 100000000 & 0 & 0.3 & 0 & 30000 & 0 & 1000 \\
\hline 100000000 & 4 & 0.6 & 0.15 & 40000 & 10000 & 1500 \\
\hline 100000000 & 7 & 0.35 & 0.05 & 70000 & 10000 & 500 \\
\hline 400000000 & 0 & 0.75 & 0 & 60000 & 0 & 5000 \\
\hline 25000000 & 7 & 1.75 & 0.25 & 35000 & 5000 & 1250 \\
\hline$\sum_{i=1}^{17} I_{D}^{2}=$ & $\sum_{i=1}^{17}$ & 22.15 & 2.15 & $\begin{array}{l}\sum_{i=1}^{17} A . I_{\mathbf{D}} \\
1524400\end{array}$ & $\sum_{i=1}^{17} h$ & $\sum_{i=1}^{17}$ \\
\hline
\end{tabular}

Table 4: Multiplication Results of the Figures in Table 1 above (Continuation)

\begin{tabular}{|c|c|c|c|c|c|}
\hline $\mathbf{A} / \mathbf{I}_{\mathbf{T}}$ & $\mathbf{E} / \mathbf{I}_{\mathbf{T}}$ & $\mathbf{D} / \mathbf{I}_{\mathbf{T}}$ & $\mathbf{1} / \mathbf{I}_{\mathbf{T}}$ & $\mathbf{I}_{\mathbf{D}} \mathbf{I}_{\mathbf{T}}$ & $\left(\mathbf{1} / \mathbf{I}_{\mathbf{T}}\right)^{2}$ \\
\hline $3.6364 \mathrm{E}-05$ & 0 & $9.09091 \mathrm{E}-07$ & $9.09091 \mathrm{E}-06$ & 0.181818182 & $8.26446 \mathrm{E}-11$ \\
\hline 0.00023077 & 0 & $3.84615 \mathrm{E}-05$ & $7.69231 \mathrm{E}-05$ & 0.076923077 & $5.91716 \mathrm{E}-09$ \\
\hline 0.00034783 & $4.34783 \mathrm{E}-05$ & $1.73913 \mathrm{E}-05$ & $4.34783 \mathrm{E}-05$ & 0.565217391 & $1.89036 \mathrm{E}-09$ \\
\hline $3.8462 \mathrm{E}-05$ & $7.69231 \mathrm{E}-06$ & $7.69231 \mathrm{E}-07$ & $7.69231 \mathrm{E}-06$ & 0.076923077 & $5.91716 \mathrm{E}-11$ \\
\hline 0.00052174 & $4.34783 \mathrm{E}-05$ & $8.69565 \mathrm{E}-06$ & $4.34783 \mathrm{E}-05$ & 0.304347826 & $1.89036 \mathrm{E}-09$ \\
\hline 0.00031746 & $3.96825 \mathrm{E}-05$ & $1.5873 \mathrm{E}-05$ & $3.96825 \mathrm{E}-05$ & 0.984126984 & $1.5747 \mathrm{E}-09$ \\
\hline 0.00010526 & $2.63158 \mathrm{E}-05$ & $7.89474 \mathrm{E}-06$ & $2.63158 \mathrm{E}-05$ & 0.052631579 & $6.92521 \mathrm{E}-10$ \\
\hline 0.00051852 & $3.7037 \mathrm{E}-05$ & $7.40741 \mathrm{E}-06$ & $3.7037 \mathrm{E}-05$ & 0.481481481 & $1.37174 \mathrm{E}-09$ \\
\hline $9.5238 \mathrm{E}-05$ & $9.52381 \mathrm{E}-06$ & $9.52381 \mathrm{E}-07$ & $9.52381 \mathrm{E}-06$ & 0.142857143 & $9.07029 \mathrm{E}-11$ \\
\hline 0.00005 & 0 & $4.16667 \mathrm{E}-06$ & $1.66667 \mathrm{E}-05$ & 0.916666667 & $2.77778 \mathrm{E}-10$ \\
\hline 0.00015556 & 0 & $3.33333 \mathrm{E}-06$ & $2.22222 \mathrm{E}-05$ & 0.333333333 & $4.93827 \mathrm{E}-10$ \\
\hline $3.8095 \mathrm{E}-05$ & 0 & $2.38095 \mathrm{E}-07$ & $4.7619 \mathrm{E}-06$ & 0.095238095 & $2.26757 \mathrm{E}-11$ \\
\hline $3.5294 \mathrm{E}-05$ & 0 & $1.17647 \mathrm{E}-06$ & $1.17647 \mathrm{E}-05$ & 0.117647059 & $1.38408 \mathrm{E}-10$ \\
\hline 0.00008 & 0.00002 & 0.000003 & 0.00002 & 0.2 & $4 \mathrm{E}-10$ \\
\hline 0.0001 & $1.42857 \mathrm{E}-05$ & $7.14286 \mathrm{E}-07$ & $1.42857 \mathrm{E}-05$ & 0.142857143 & $2.04082 \mathrm{E}-10$ \\
\hline 0.00005 & 0 & $4.16667 \mathrm{E}-06$ & $1.66667 \mathrm{E}-05$ & 0.333333333 & $2.77778 \mathrm{E}-10$ \\
\hline 0.0002 & $2.85714 \mathrm{E}-05$ & $7.14286 \mathrm{E}-06$ & $2.85714 \mathrm{E}-05$ & 0.142857143 & $8.16327 \mathrm{E}-10$ \\
\hline 17 & 17 & 17 & 17 & 17 & 17 \\
$\sum_{i=1} \mathbf{A} / I_{T}=$ & $\sum_{i=1} \mathbf{E} / I_{T}=$ & $\sum_{i=1} D / I_{T}=$ & $\sum_{i=1} 1 / I_{T}=$ & $\sum_{i=1} / I_{T}=$ & $\sum_{i=1}\left(1 / I_{T}\right)^{2}=$ \\
0.00292058 & 0.000270065 & 0.000122293 & 0.000428161 & 5.148259514 & $1.62002 \mathrm{E}-08$ \\
\end{tabular}




$$
\begin{gathered}
\sum_{i=1}^{17} \mathbf{A}=110, \quad \sum_{i=1}^{17} E=10, \quad \sum_{i=1}^{17} I_{D}=250800, \quad \sum_{i=1}^{17} I_{T}=11092000, \\
\sum_{i=1}^{17} \mathbf{A}^{2}=888, \quad \sum_{i=1}^{17} E^{2}=10, \quad \sum_{i=1}^{17} I_{D}^{2}=6107040000, \quad \sum_{i=1}^{17} A . E=79, \quad \sum_{i=1}^{17} A . D=22.15
\end{gathered},
$$

Hence, equations (18), (19), (20), (21), and (22) correspondingly become,

$$
\begin{gathered}
888 x+79 y+1524400 z+0.29206 e-2 u+110 v=22.15 \\
79 x+10 y+109800 z+0.27007 e-3 u+10 v=2.15 \\
1524400 x+109800 y+6107040000 z+5.14825951 u+250800 v=50970 \\
0.29206 e-2 x+0.27007 e-3 y+5.1482595 z+0.1620002 e-7 u+0.42816 e-3 v=0.12229 e-3 \\
110 x+10 y+250800 z+0.42816 e-3 u+17 v=3.55
\end{gathered}
$$

Therefore, solving equations (23) - (27) simultaneously yields;

$\mathrm{x}=-0.01375092400$

$\mathrm{y}=0.06120630870$

$\mathrm{z}=0.000003178833528$

$\mathrm{u}=6931.544134$

$\mathrm{v}=0.04032218636$

Also putting the values of $\mathrm{x}, \mathrm{y}, \mathrm{z}, \mathrm{u}$ and $\mathrm{v}$ into equation (11) gives,

$$
\mathrm{D}=-0.01375092400 \mathrm{~A}+0.06120630870 \mathrm{E}+0.000003178833528 \mathrm{ID}+0.04032218636
$$

Consequently, equation (28) is the developed model for predicting divorce possibility in marriages in Taraba state.

\section{DISCUSSION OF RESULTS}

\subsection{Optimization of the Model}

Linear optimization is a mathematical method for defining a way to achieve the best result in a given Mathematical model, for some list of condition characterized as linear relationship. And more strictly, optimization is a process for programming an objective function, subject to some equation or inequality constraints. For this reason, we observed that at turning point, $\mathrm{D}=0$ (which means that the point of hope for any marriage in Nigeria is when Divorce rate, $\mathrm{D}=0$. Since Divorce rate cannot be negative any value higher than zero is an indication worth taking note or watching out for.

\subsection{Observation on the Nature of the Model's Extreme Values}

This stage is necessary to ascertain whether the model's extreme point is minimum, maximum or a saddle.

Generally, a given function $\mathrm{f}(\mathrm{x}, \mathrm{y})$ that obeys the continuity of partial derivatives as we let;

$$
\mathrm{A}=\frac{\partial^{2} f}{\partial x^{2}} ; \quad \mathrm{B}=\frac{\partial^{2} f}{\partial x \partial y} ; \quad \mathrm{C}=\frac{\partial^{2} f}{\partial y^{2}} \text { then, }
$$

1. If $B^{2}-A C<0$, then $f(x, y)$, has extreme value at $\left(x_{0}, y_{0}\right)$ and minimum if $A>0$ and it is maximum if $A<0$.

2. If $B^{2}-A C>0$ or $A B<C^{2}$ then, $f(x, y)$ has no extreme value, that is, it has a saddle point at $\left(x_{0,}, y_{0}\right)$.

3. If $B^{2}-A C=0$, then no information is derivable about the functions' extreme values. 
Similarly, from our model equation (11),

$$
D=x A+y E+z I_{D}+\frac{u}{I_{T}}+v
$$

Recall that from (3) $D=k_{3} I_{D}$

$$
\Rightarrow \quad I_{D}=\frac{D}{k_{3}}
$$

Similarly, to obtain the extreme point, we use equation (11) to get two unknown. To achieve that, let's consider adding the equation (1), (2), and (3) together from chapter three as,

$$
3 D=k_{1} A+k_{2} E+k_{3} I_{D}
$$

Putting (29) into (30) yields;

$$
\begin{aligned}
& 3 D=k_{1} A+k_{2} E+D \\
& \Rightarrow \quad 2 D=k_{1} A+k_{2} E \\
& \Rightarrow \quad E=\frac{2 D-k_{1} A}{k_{2}} \\
& D=x A+y E+z\left(\frac{D}{k_{3}}\right)+\frac{u}{I_{T}}+v \\
& D=x A+y\left[\frac{2 D-k_{1} A}{k_{2}}\right]+z\left(\frac{D}{k_{3}}\right)+\frac{u}{I_{T}}+v \\
& D=x A+\frac{2 y D}{k_{2}}-\frac{k_{1} y A}{k_{2}}+\frac{z D}{k_{3}}+\frac{u}{I_{T}}+v \\
& D-y D-\frac{z D}{k_{3}}=\left(x-\frac{k_{1}}{2}\right) A+\frac{u}{I_{T}}+v \\
& \left(1-y-\frac{z}{k_{3}}\right) D=\left(x-\frac{k_{1}}{2}\right) A+\frac{u}{I_{T}} v \\
& \Rightarrow \quad D=\left(\frac{x k_{2}-k_{1} y}{k_{2}}\right)\left(\frac{k_{2} k_{3}}{k_{2} k_{3}-2 y k_{3}-z k_{2}}\right) A+\left(\frac{u}{I_{T}}\right)\left(\frac{k_{2} k_{3}}{k_{2} k_{3}-2 y k_{3}-z k_{2}}\right)+\frac{k_{2} k_{3} v}{k_{2} k_{3}-2 y k_{3}-z k_{2}} \\
& \Rightarrow \quad D=\left(\frac{k_{3}\left(x k_{2}-k_{1} y\right)}{k_{2} k_{3}-2 y k_{3}-z k_{2}}\right) A+\frac{\left(k_{2} k_{3}\right) u}{\left(k_{2} k_{3}-2 y k_{3}-z k_{2}\right) I_{T}}+\frac{k_{2} k_{3} v}{k_{2} k_{3}-2 y k_{3}-z k_{2}}
\end{aligned}
$$

Meanwhile, differentiating (32) partially with respect to $A$ and $I_{D}$ gives,

Similarly,

$$
\begin{gathered}
D=\left[\frac{k_{3}\left(2 x-k_{1}\right)}{k_{3}-k y-z}\right] A+\frac{u k_{3}}{\left(k_{3}-k y-z\right) I_{T}}+\frac{k_{3} v}{k_{3}-k y-z} \\
\frac{\partial D}{\partial A}=\frac{k_{3}\left(x k_{2}-k_{1} y\right)}{k_{2} k_{3}-2 y k_{3}-z k_{2}}, \\
\frac{\partial^{2} D}{\partial A^{2}}=0
\end{gathered}
$$

$$
\frac{\partial D}{\partial I_{T}}=\frac{-\left(k_{2} k_{3}\right) u}{\left(k_{2} k_{3}-2 y k_{3}-z k_{2}\right) I_{T}^{2}},
$$


Also,

$$
\frac{\partial^{2} D}{\partial I_{T}^{2}}=\frac{-\left(k_{2} k_{3}\right) u}{\left(k_{2} k_{3}-2 y k_{3}-z k_{2}\right) I_{T}^{3}}
$$

$$
\frac{\partial^{2} D}{\partial A \partial I_{T}}=\frac{\partial}{\partial A}\left(\frac{-\left(k_{2} k_{3}\right) u}{\left(k_{2} k_{3}-2 y k_{3}-z k_{2}\right) I_{T}^{3}}\right)=0
$$

Assuming, $A=\frac{\partial^{2} D}{\partial A^{2}}, \mathrm{~B}=\frac{\partial^{2} D}{\partial A \partial I_{T}}$ and $\mathrm{C}=\frac{\partial^{2} D}{\partial I_{T}^{2}}$, by substituting our critical values, then,

$$
B^{2}-A C=0-\left(\frac{-\left(k_{2} k_{3}\right) u}{\left(k_{2} k_{3}-2 y k_{3}-z k_{2}\right) I_{T}^{3}}\right)(0)
$$

Therefore,

$$
B^{2}-A C=0
$$

(It means that, the optimal/extreme value is saddle since $\left(B^{2}-A C=0\right)$.

Remark: From our optimal result in equation (37) above, we agree that there is neither maximum nor minimum level of divorce tendency in all Nigeria marriages. This means that divorce tendency of a marriage can take values close to $0 \%$ and even as high as $100 \%$.

\subsection{Validation of the Model}

After the model became ready, we carried out a pilot test using the data given in various parts of the area in consideration for the research to see whether the model conform to reality. And the validation test of the model is buttressed in the tables of figures below.

Table 5. Table for the Validation of the Model

\begin{tabular}{|c|c|c|c|}
\hline $\begin{array}{c}\text { Questionnaires data on } \\
\text { Divorce }\left(\mathrm{D}_{\mathrm{O}}\right)\end{array}$ & $\begin{array}{c}\text { Model Predictions on Divorce } \\
(\mathrm{D})\end{array}$ & Absolute Error & Percentage Difference \\
\hline 0.1 & 0.111909199 & 0.011909199 & 1.19091985 \\
\hline 0.5 & 0.535443951 & 0.035443951 & 3.54439505 \\
\hline 0.4 & 0.334217423 & 0.065782577 & 6.578257699 \\
\hline 0.1 & 0.117881781 & 0.017881781 & 1.78817806 \\
\hline 0.2 & 0.260140726 & 0.060140726 & 6.014072584 \\
\hline 0.4 & 0.34541745 & 0.05458255 & 5.458255029 \\
\hline 0.3 & 0.235291522 & 0.064708478 & 6.470847773 \\
\hline 0.2 & 0.207064252 & 0.007064252 & 0.706425174 \\
\hline 0.1 & 0.077716464 & 0.022283536 & 2.228353598 \\
\hline 0.25 & 0.289430994 & 0.039430994 & 3.943099397 \\
\hline 0.15 & 0.145782535 & 0.004217465 & 0.421746463 \\
\hline 0.05 & 0.026898818 & 0.023101182 & 2.310118206 \\
\hline 0.1 & 0.112405328 & 0.012405328 & 1.240532769 \\
\hline 0.15 & 0.216944017 & 0.066944017 & 6.694401702 \\
\hline 0.05 & 0.136082421 & 0.086082421 & 8.60824214 \\
\hline 0.25 & 0.17817182 & 0.07182818 & 7.182817951 \\
\hline 0.25 & 0.219210313 & 0.030789687 & 3.078968719 \\
\hline
\end{tabular}

Remarks: it is clear from the Table 5 above that our real life gathered data $\left({ }^{D_{Q}}\right)$ on marriage Divorce Tendency is approximately coincided with the model results $\left(D_{M}\right)$ as shown. The results for $D_{M}$ is obtained by substituting each respondent data in table1 into equation (28). Similarly, observations from the difference between the two results therefore suggest how high our developed model is performing and thus recommend the model as a standard measurement for computing the rate of marriage Divorce Tendency in Nigeria. 


\subsection{Correlation Coefficient between Gathered Data and Model Predictions for Divorce rate in Taraba State, Nigeria}

For benefit of the model's correlation we generate a new table from table 5.above as given beneath,

Table 6: Values to Test for Model correlation

\begin{tabular}{|c|c|c|c|c|}
\hline $\begin{array}{l}\text { Questionnaires data on } \\
\text { Divorce }\left(\mathrm{D}_{\mathrm{Q}}\right)\end{array}$ & $\begin{array}{l}\text { Model Predictionson } \\
\text { Divorce D }\end{array}$ & $\mathrm{D}_{\mathrm{Q}}^{2}$ & $\mathrm{D}^{2}$ & $\mathrm{D}_{\mathrm{Q}}{ }^{*} \mathrm{D}$ \\
\hline 0.1 & 0.111909199 & 0.01 & 0.01252367 & 0.01119092 \\
\hline 0.5 & 0.535443951 & 0.25 & 0.28670022 & 0.267721976 \\
\hline 0.4 & 0.334217423 & 0.16 & 0.11170129 & 0.133686969 \\
\hline 0.1 & 0.117881781 & 0.01 & 0.01389611 & 0.011788178 \\
\hline 0.2 & 0.260140726 & 0.04 & 0.0676732 & 0.052028145 \\
\hline 0.4 & 0.34541745 & 0.16 & 0.11931321 & 0.13816698 \\
\hline 0.3 & 0.235291522 & 0.09 & 0.0553621 & 0.070587457 \\
\hline 0.2 & 0.207064252 & 0.04 & 0.0428756 & 0.04141285 \\
\hline 0.1 & 0.077716464 & 0.01 & 0.00603985 & 0.007771646 \\
\hline 0.25 & 0.289430994 & 0.0625 & 0.0837703 & 0.072357749 \\
\hline 0.15 & 0.145782535 & 0.0225 & 0.02125255 & 0.02186738 \\
\hline 0.05 & 0.026898818 & 0.0025 & 0.00072355 & 0.001344941 \\
\hline 0.1 & 0.112405328 & 0.01 & 0.01263496 & 0.011240533 \\
\hline 0.15 & 0.216944017 & 0.0225 & 0.04706471 & 0.032541603 \\
\hline 0.05 & 0.136082421 & 0.0025 & 0.01851843 & 0.006804121 \\
\hline 0.25 & 0.17817182 & 0.0625 & 0.0317452 & 0.044542955 \\
\hline $0.25 \mathrm{e}$ & 0.219210313 & 0.0625 & 0.04805316 & 0.054802578 \\
\hline$=3.55$ & $\sum_{3.550009014} D=$ & & $\sum_{0.9798481} \mathrm{D}_{\mathrm{Q}}{ }^{2}=$ & $\sum_{=0.979856981} \mathrm{D}^{*} \mathrm{D}_{\mathrm{Q}}$ \\
\hline
\end{tabular}

Letting $\mathrm{D}_{Q}=X$ and $D=Y$, using the correlation formula below, we have;

$$
\text { Correlation Coefficient } \mathrm{r}=\frac{n \sum X Y-\sum X \sum Y}{\sqrt{\left.\left[n \sum X^{2}-\left(\sum X\right)^{2}\right] n \sum Y^{2}-\left(\sum Y\right)^{2}\right]}}
$$

But for this study, $\quad \mathrm{n}=17$ (number of respondents)

$$
\begin{aligned}
& \sum X=3.55, \sum Y=3.550009014, \sum X^{2}=1.0175, \sum Y^{2}=0.9798481 \text { and } \\
& \sum X Y=0.979856981 . \\
& \therefore r=\frac{(17 \times 0.97985681)-(3.55 \times 3.550009014)}{\left.\sqrt{\left[17(1.0175)-(3.55)^{2}\right]\left[17(0.9798481)-(3.550009014)^{2}\right.}\right]} \\
& \mathrm{r}=\frac{4.05503377}{\sqrt{(4.695)(4.054853701)}} \Rightarrow \quad \mathrm{r}=\frac{4.05503377}{4.363202737}
\end{aligned}
$$

Therefore,

$$
r=0.929370926 \quad \text { or } \quad r=92.94 \%
$$

Remarks: from the correlation coefficient analysis made using the results in table 6., it was observed that that the correlation coefficient denoted by (r), is $r=0.929370926$. From now, we have determined that our model has approximately $92.94 \%$ of correlation from the real life data employed. Categorically, it implies there is a high degree ofcorrespondence, as a result, recommending the model as an accepted measure for estimating occurrence of divorce anytime. 


\subsection{Graphical Comparison of the Real Life Data and the Model Estimated Data on Divorce}

In this section ofanalysis, computational assessment of the data and the model predicted data was madevia plotting of graph to see the authentication and alignment of the questionnaire data and the model results as shown below;

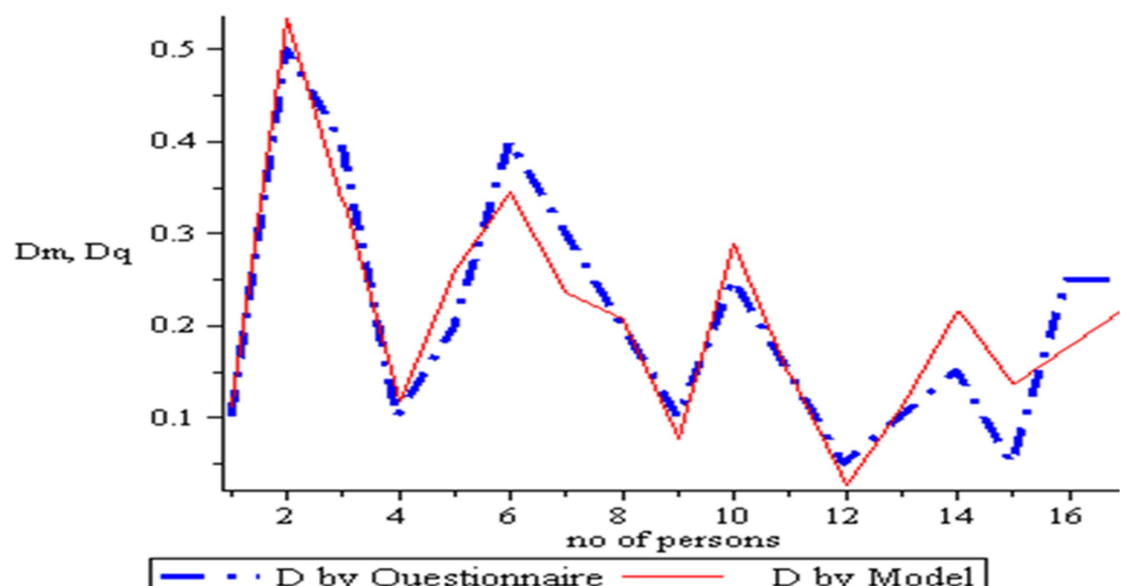

Fig1: Graph showing Relationship between Persons and their Divorce Tendencies obtained by our model (Dm) Data (Dq)

\section{SUMMARY AND CONCLUSIONS}

\subsection{Summary of Findings}

The model validation test in section 4.3 confirmed that the model is appropriate for the would-be users of the model since the absolute difference between the plotted model data predictions and the questionnaire data is very minimal. And the difference is in fact approximately less than 0.07 units (as confirmed by table 5. above). The little error why the model did not give exact solution as the gathered data nonetheless, is as a result of the fact that no marriage, no matter how good it may be viewed from the outward, is totally free of all the factors promoting divorce in marriages.

In this research work, the system under consideration is divorce in marriage. In the meantime, based on the focus of this study, a collection of data from our area of interest for both stable and faulty marriages was made. And the data were utilized to validate our formulated model. Finally, the model validation result was used to test if actually our modelconforms to reality or not. But, from the correlation coefficient breakdown in Table6.,our model has $92.94 \%$ of correlation to the real life data exploited. Tables (5), (6) and the graph in figure (4.1) were employed to give brief understanding of the model validation and application.

Based on the summary attained by this study, the equation (28) gives the proposed model equation for predicting the divorce rate of couples in marriage institution. With it when any married person(s) input or substitutes his/her data accurately into the formulated model formula in the equation, the estimated percentage value for their divorce rate would be estimated.

\subsection{Conclusion}

Based on the findings in the result discussion above, equation (28) gives the proposed model equation for predicting the divorce rate of couples in marriage institutions. With the developed model, any married person(s) who inputs or substitutes his/her required data accurately into the formulated model in equation (28) is assured of obtaining an estimated percentage value for what their divorce rate would be.

Equally, when the model became ready, it was tested by comparing its computed data outcome with the real life data collected as shown in Table 1. The outcome of the comparison showed that our model is in strong conformity to reality values. This assertion was confirmed with the correlation coefficient of approximately $92.94 \%$ of agreement with real life data.

Likewise, from all levels of our analysis from the questionnaire results in sections 3, 4 and 5; it is obvious that the tendency for divorce is highly dependent on thecouples age difference, educational difference, income difference and their total income as a family. The results from equation (37) therefore indicated that, there is neither a maximum nor minimumdivorce rate that any set of couples could attain, and still escape divorce. It thus means that, couples with very low or negligible divorce rate and higher divorce rates are both in the same danger of breaking up if they fail to work it out. This is because, there may be gifts and fruits of the Holy Spirit but there is no gift of good marriage guaranteed in the scriptures.Meanwhile, at any point, couples are advised to be extremely careful in order to sustain their marriages from 
divorce. This is because a shift from blissful moment in marriage could mean the marriage is heading for a divorce. Finally, our optimization result in section 4 , indicates that the only point or moment couples can relax or be free from divorce is when their $\mathrm{D}=0 \%$.

\section{References}

[1] M. Buchibrand, 7 forms of divorce in Africa, Retrieved on 22nd May 2018 from https://www.nairaland.com/2526539/7-formsdivorce-africa, 2016.

[2] N. Em, National Bureau of Statistics reveal low divorce rate in Nigeria. Article on marriage. Retrieved on 15th June, 2018, from https://www.naija.ng/887623-must-read-based-stats-one-thing-nigerians-rarely.html\#887623, 2017.

[3] G. Grath, The childless marriage an expository study of couples who do not want children. An Article on family. Retrieved on 20th June 2018.http://www.guidea.com.br/the-childless-marriage-an-exploratory-study-of-couples-who-do-not-want-children.pdf, 2001.

[4] L. A. Obiani, Social indicators and effects of marriage divorce in African societies. The Business \& Management Review, Volume 4 Number 4.4 Retrieved on the 19th June, 2018, from https://pdfs.semanticscholar.org/56fc/5403c212d56827b223b0f7c3b12fdc670e5b, 2014.

[5] L. Shawn, Informal separation; a way to save your marriage, or just delaying the inevitable. Retrieved on the 1st August, 2018 from, https://divorceandyourmoney.com/blogs/informal-separation, 2017.

[6] A. Vida, Determinants of divorce in Ghana, 2008. Retrieved on 18th June, 2018 from http://www.ugspace.ug.edu.gh/bitstream/handle/123456789/5438/Vida\%20Akuamoah_Determinants_of_divorce, 2013.

[7] The Bible: What Marriage is, Genesis 2:24, Thus it is for this cause that a man shall leave his mother and clings to his wife and together they become one, Retrieved on the 19th June 2018, from http://www.e-bible.com/kjv .

[8] The law dictionary. What is divorce? Featuring Black's Law Dictionary Free Online Legal Dictionary 2nd Ed. Retrieved on the 25th June, 2018, from https://thelawdictionary.org/divorce/.

[9] Sheilalacadin, Divorce Process: Making It Easy For The Children. Dependable Homebuyers. Retrieved on the 27th of March, 2020 from https://www.dependablehomebuyers.com/blog/divorce-process-making-it-easy-for-the-children/, 2019.

[10] P. P. Gambrah. and Y. Adzadu, Mathematical model of divorce epidemic in Ghana. International Journal of Statistics and Applied Mathematics. 2018, 3(2): 395-401,

[11] I. Duato, and L. Jódar, Mathematical modelling of the spread of divorce in Spain. Mathematical and Computer Modelling. 2013; 57: $1732-1737$

[12] R. L., Simons, K. H. Lin., L. C., Gordon, R. D. Conger, and F. O., Lorenz, Explaining the higher incidence of adjustment problems among children of divorce compared with those in two-parent families. Journal of Marriage and the Family. 1999; 61:10201034.

[13] K. N., Hipke, S. A., Wolchik, I. N. Sandler and S. L., Braver, Predictors of children's intervention-induced resilience in a parenting program for divorced mothers. Family Relations 2002; 51:121-130.

[14] E. M. Hetherington and M. Stanley-Hagen, The Adjustment of children with divorced parents: A risk and resiliency perspective. Journal of Child Psychology. 1999; 40:129-140.

[15] H. Nair and A. D. Murray, Predictors of attachment security in preschool children from intact and divorced families. Journal of Genetic Psychology. 2005; 166:245-263.

[16] N. Kalu, How to Divorce in Nigeria. Step by Step Guides, Documents and Requirements. Retrieved on the 19th June, 2018, from https://infoguidenigeria.com/divorce-nigeria-step-step-guides-documents-requirements/, 2015.

[17] Lawpadi, Grounds for divorce in Nigeria. Family and Health by Admin. Retrieved 16th June, 2018 from http://www.lawpadi.com/grounds-for-divorce-in-nigeria/, 2018.

\section{Authors' Profiles}

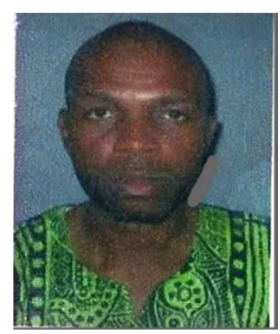

OGWUMU O.D. is currently employed as a Lecturer in the Department of Mathematics and Statistics, Faculty of Pure and Applied Science, Federal University Wukari, Nigeria. He obtained his Ph.D. from the University of Ilorin, Nigeria. He is an active researcher coupled with a vast teaching experience. He has taught various courses such as Mathematical Modelling, Mathematical Methods, Differential Equations, Optimisation Theory, Vectors and Tensor Analysis, Algebra and Trigonometry. His main research interests ranges from Mathematical Modelling, Differential Equations, optimization and control. He has published more than 35 research articles (including Text books) in reputed international journals and publishing houses.

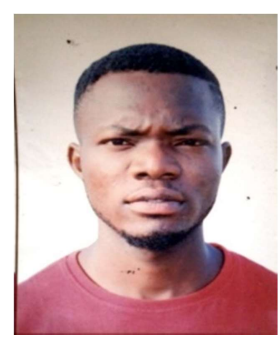

Ezeh A.T. holds a BSc Degree in Mathematics from the Department of Mathematics, Federal University Wukari, Nigeria. He is a First Class Graduate with main research interest in the area of Mathematical Modelling, Optimisation and Differential Equations. He has published some good research articles in both reputed international/national journals and publishing houses 

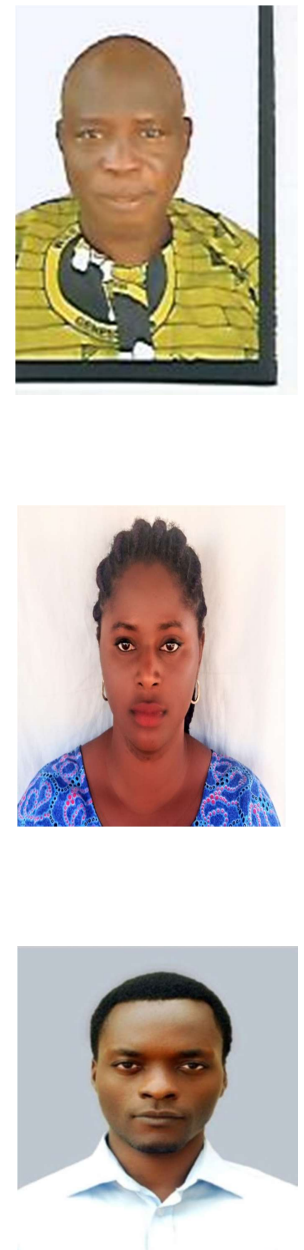

Keto, K. M. holds a BSc Degree in Mathematics from the Department of Mathematics, Federal University Wukari, Nigeria. He is one of the best researchers of his set in his undergrduate days. His main research interest is in the area of Mathematical Modelling and Differential Equations. He has published some good research articles in both reputed international/national journals and publishing houses

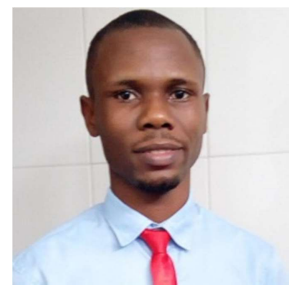

Amakoromo G.I. is a staff of Federal University Wukari, Nigeria in the Department of Mathematics and Statistics, Faculty of Pure and Applied Science. She holds a BSc. from the University of Jos Nigeria and has her MSc. (in view) from Nasarawa State Universit, Nigeria. Her research interest is in the area of Mathematical Modelling and Differential Equations. She has published some good research articles in both reputed international/national journals and publishing houses

Ogofotha M. O. holds a BSc Degree in Mathematics from the Department of Mathematics, Federal University Wukari, Nigeria. He is one of the best students of his set in his undergrduate days. His main research interest is in the area of Mathematical Modelling and Differential Equations. He has published some good research articles in both reputed international/national journals and publishing houses

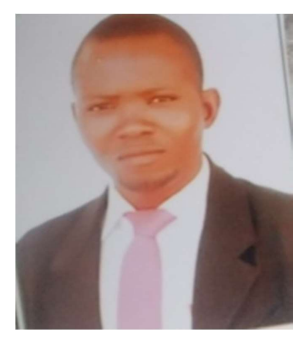

Elugah J.I. holds a BSc Degree in Mathematics from the Department of Mathematics, Federal University Wukari, Nigeria. He is one of the best students of his set in his undergrduate days. His main research interest is in the area of Fluid Mechanics and Differential Equations. He has published some good research articles in both reputed international/national journals and publishing houses

How to cite this paper: Ogwumu Onah.David, Kyagya T.Yusuf, Amakoromo Grace.I., Keto, Kingsley M., Ezeh A.Tochukwu., Ogofotha Marvellous O., Elugah Joseph.I.. " A Mathematical Model for Predicting Rate of Divorce Tendency in Nigeria: A Study of Taraba State, Nigeria ", International Journal of Mathematical Sciences and Computing (IJMSC), Vol.6, No.5, pp.15-28, 2020. DOI: 10.5815/IJMSC.2020.05.02 